\title{
Origins Space Telescope: the far infrared imager and polarimeter FIP
}

Johannes Staguhn, Edward Amatucci, Lee Armus, Damon Bradley, Ruth Carter, et al.

Johannes Staguhn, Edward Amatucci, Lee Armus, Damon Bradley, Ruth Carter, David Chuss, James Corsetti, Asantha Cooray, Joseph Howard, David Leisawitz, Margaret Meixner, Samuel H. Moseley, Alexandra Pope, Joaquin Vieira, Edward Wollack, "Origins Space Telescope: the far infrared imager and polarimeter FIP," Proc. SPIE 10698, Space Telescopes and Instrumentation 2018: Optical, Infrared, and Millimeter Wave, 106981A (30 July 2018); doi: 10.1117/12.2312626

Event: SPIE Astronomical Telescopes + Instrumentation, 2018, Austin, Texas, United States 


\title{
Origins Space Telescope: The Far Infrared Imager and Polarimeter FIP
}

Johannes Staguhn ${ }^{\mathrm{a}, \mathrm{b}}$, Edward Amatucci ${ }^{\mathrm{b}}$, Lee Armus ${ }^{\mathrm{c}}$, Damon Bradley ${ }^{\mathrm{b}}$, Ruth Carter ${ }^{\mathrm{b}}$, David Chuss $^{\mathrm{d}}$, James Corsetti ${ }^{\mathrm{b}}$, Asantha Cooray ${ }^{\mathrm{e}}$, Joseph Howard ${ }^{\mathrm{b}}$, David Leisawitz ${ }^{\mathrm{b}}$, Margaret Meixner ${ }^{\mathrm{f}}$, Samuel H. Moseley ${ }^{\mathrm{b}}$, Alexandra Pope ${ }^{\mathrm{g}}$, Joacquin Vierra ${ }^{\mathrm{h}}$, and Edward Wollack ${ }^{\mathrm{b}}$

a Johns Hopkins University, Baltimore, Dpt. of Physics \& Astronomy, MD 21218, USA

${ }^{\mathrm{b} N A S A}$ Goddard Space Flight Center, Greenbelt, MD 20771, USA

${ }^{\mathrm{c}}$ IPAC, Caltech, Pasadena, CA 91125, USA

${ }^{d}$ Villa Nova University, 800 Lancaster Avenue, Villanova, PA 19085, USA

eDepartment of Physics \& Astronomy, University of California, Irvine, 92697, USA

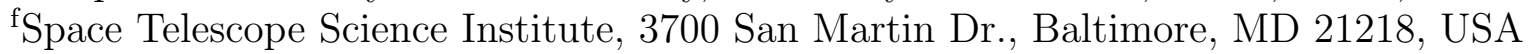

' University of Massachusetts, Amherst, MA 01003-9305, USA

${ }^{\text {h} U n i v e r s i t y ~ o f ~ I l l i n o i s, ~} 229$ Astronomy, 1002 W. Green Street, Urbana IL 61801, USA

\begin{abstract}
The Origins Space Telescope (OST) is the mission concept for the Far-Infrared Surveyor, one of the four science and technology definition studies of NASA Headquarters for the 2020 Astronomy and Astrophysics Decadal survey. "Concept-1" is a cold (4 K) 9 m space telescope with five instruments, while "concept 2 " consists of a cold $5.9 \mathrm{~m}$ telescope and four instruments, providing imaging and spectroscopic capabilities between $5 \mu \mathrm{m}$ and $600 \mu \mathrm{m}$. The sensitivity provided by the observatory will be a three to four orders of magnitude improvement over currently achieved observational capabilities, allowing to address a wide range of new and so far inaccessible scientific questions, ranging from bio-signatures in the atmospheres of exo-planets to the production of the first metals in the universe right after the end of re-ionization.

Here we present the Far Infrared Imager and Polarimeter (FIP) for OST. The camera will cover four bands, $50 \mu \mathrm{m}, 100 \mu \mathrm{m}, 250 \mu \mathrm{m}$, and $500 \mu \mathrm{m}$. In the "concept 1" version of the instrument, FIP will allow for differential polarimetry with the ability to observe two colors simultaneously, while all four bands can be observed simultaneously in total power mode. The confusion limit in the total power mode will be reached in only $8 \mathrm{~ms}$ at $500 \mu \mathrm{m}$, while at $50 \mu \mathrm{m}$ the source density in the sky is so low that at OST's angular resolution of $\sim 2$ " in this band the source confusion limit will only be reached after about two hours of integration with the "concept-2" version of FIP ("concept-1" FIP will not be confusion limited at $50 \mu \mathrm{m}$, no matter how long it integrates). Science topics that can be addressed by the camera include, but are not limited to, galactic and extragalactic magnetic field studies, deep galaxy surveys, and outer Solar System objects.
\end{abstract}

Keywords: far-infrared, space observatories, cryogenic detectors, far-infrared instruments

\section{INTRODUCTION}

The Far-Infrared (FIR) foreground level in space, which is dominated by Zodiacal and Galactic emission, is very low. Only at the longer wavelengths $>300 \mu \mathrm{m}$ does the Cosmic Microwave Emission begin to contribute significantly as a background source. Fig. 1 shows the Noise Equivalent sky foreground Power for a fractional bandwidth $R=3.3$, typical for continuum cameras, and for a fractional bandwidth of $R=1,500$, typical for spectroscopy (with noise bandwidth equivalent to the spectral channel resolution, e.g. a grating spectrometer). In order to be dominated by these foregrounds, space based FIR observatories need to be cooled to cryogenic

Further author information: (Send correspondence to Johannes Staguhn)

E-mail: johannes.staguhn@nasa.gov, Telephone: 13018147840

Space Telescopes and Instrumentation 2018: Optical, Infrared, and Millimeter Wave, edited by Makenzie Lystrup, Howard A. MacEwen, Giovanni G. Fazio, Proc. of SPIE Vol. 10698, 106981A

(c) 2018 SPIE · CCC code: 0277-786X/18/\$18 - doi: 10.1117/12.2312626 
temperatures of $\sim 4 K$. Indeed the baseline for the The Origins Space Telescope (OST) reflects this: It is a cryogenic $(T=4 \mathrm{~K})$ telescope providing for background limited continuum and spectroscopy. FIP for OST will require detectors with a Noise Equivalent Power (NEP) of a few $10^{-19} \mathrm{~W} / \sqrt{\mathrm{Hz}}$, while spectrometers with $R \sim 300$ will need detectors with one order of magnitude lower NEPs, i.e. of a few $10^{-20} \mathrm{~W} / \sqrt{\mathrm{Hz}}$. Note that these numbers are independent of the telescope diameter. "Concept-1" for OST consists of a cold $9 \mathrm{~m}$ space telescope with five instruments, while "concept-2" consists of a cold $5.9 \mathrm{~m}$ telescope and four instruments, both providing a wavelength coverage between $5 \mu \mathrm{m}$ to $600 \mu \mathrm{m}$. In the following we describe OST's Far Infrared Imager and Polarimeter, FIP.

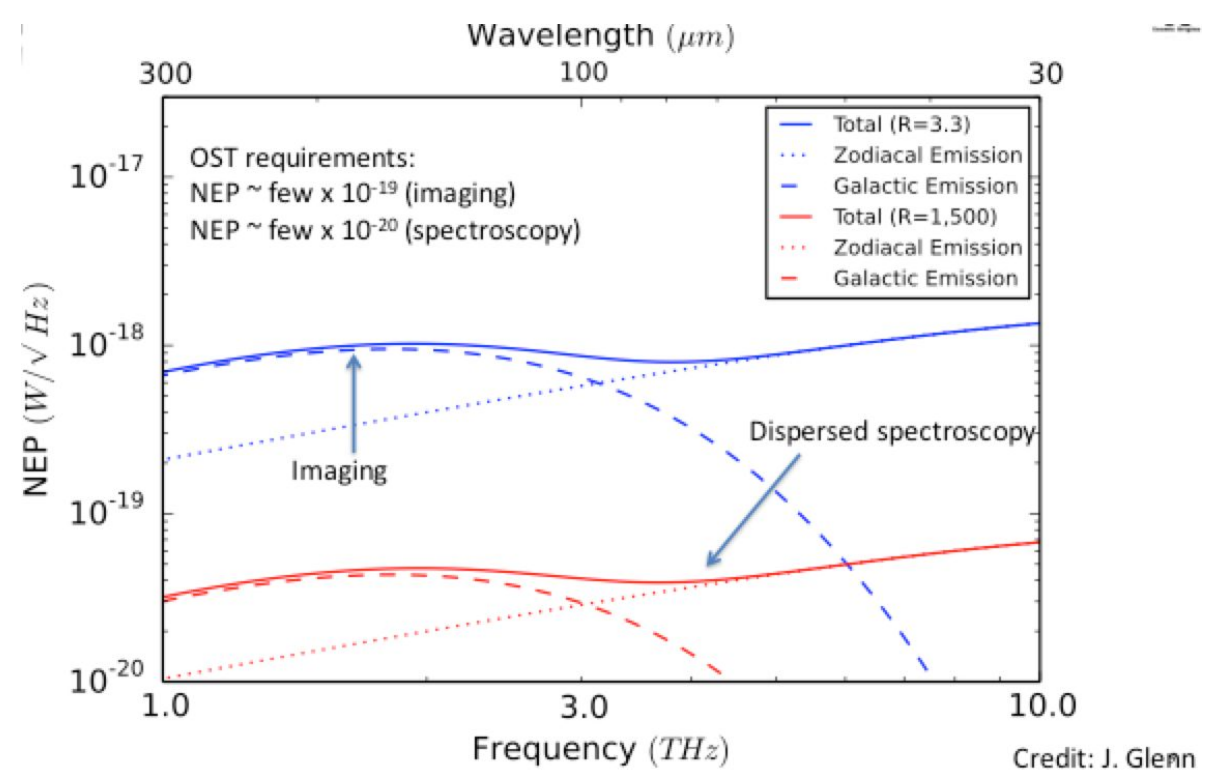

Figure 1. Sky foreground NEP for continuum (blue, $\mathrm{R}=3.3$ ) and spectroscopy (red, $\mathrm{R}=1,500$ ) which is the background for cooled $(4 \mathrm{~K})$ telescope in space in, such as OST (the numbers shown are not corrected for optical efficiencies or residual backgrounds from the observatory; Image credit: Jason Glenn).

\section{FIP SCIENCE}

FIP on OST enables large surveys of far-infrared continuum emission, surveys of solid bodies in the outer solar system, and measurements of galactic and extragalactic dust polarization, yielding information about the direction and strength of interstellar magnetic fields. With astrophysical background-limited sensitivity and angular resolution unprecedented for a far-IR telescope, FIP on OST enables studies of the first dust in the universe, properties of the dust and magnetic fields in our own and nearby galaxies, and probes the origins of our solar system with unprecedented sensitivities.

\section{INSTRUMENT DESCRIPTION}

FIP enables large-area survey imaging in four far-IR wavelength bands ranging from 50 to $500 \mu \mathrm{m}$. The camera has two operational modes, total power and polarimetry mode. In Total Power Mode, FIP quickly reaches the confusion limit at longer wavelengths $(8 \mathrm{~ms}$ at $500 \mu \mathrm{m} ; 0.4 \mathrm{~s}$ at $100 \mu \mathrm{m})$, but allows integrations with unprecedented depth at $50 \mu \mathrm{m}$, where if reaches the confusion limit after about 2 hours of integration. The FIP team optimized the instrument design to meet the maximum achievable optical efficiency in each band and to provide simultaneous wavelength coverage, while requiring only a moderate FOV from the telescope. In the "concept 1" incarnation, in Total Power Mode, FIP observes in four bands (50, 100, 250, 500 $\mu$ ) simultaneously, while in polarimetry mode, FIP operates as a differential polarimeter, simultaneously covering two bands $(50$ and $100 \mu$ or 250 and $500 \mu$ ). FIP provides diffraction-limited images with an angular resolution of $5 \times \lambda / 100 \mu \mathrm{m}$ [arcsec] and covers an instantaneous FOV of 3.0 arcmin x 4.5 arcmin at 50 and $100 \mu \mathrm{m}$ and $9.0 \operatorname{arcmin} \times 13.5$ arcmin at 
120 and $240 \mu \mathrm{m}$. In the "concept-1" configuration FIP has two orthogonal arms, each with one blue and one red channel. Light between the two arms is split into the two observing modes. In Total Power Mode, a dichroic separates the incoming radiation into a "short band" and a long band. In Polarization Mode, a half-wave plate modulator is brought into the beam and the dichroic is replaced by a polarizer. One optical arm then receives the horizontal $(\mathrm{h})$ polarized radiation, while the other receives the vertical (v) polarized radiation. The red and blue channels in both arms are then identical (either long (l) or short (s)), enabling differential polarimetry simultaneously in two bands. The center band frequencies are chosen, so each detector operates in resonance at nearly $100 \%$ optical efficiency in all frequency bands. "Concept 2" has only one arm with two detector arrays, allowing the simultaneous observation of two bands (either 50 and 100 or 250 and $500 \mu \mathrm{m}$ ) in both total power mode or polarimetry mode, while the latter is not differential, i.e. only one polarization will be measured.

\section{OPTICAL DESIGN}

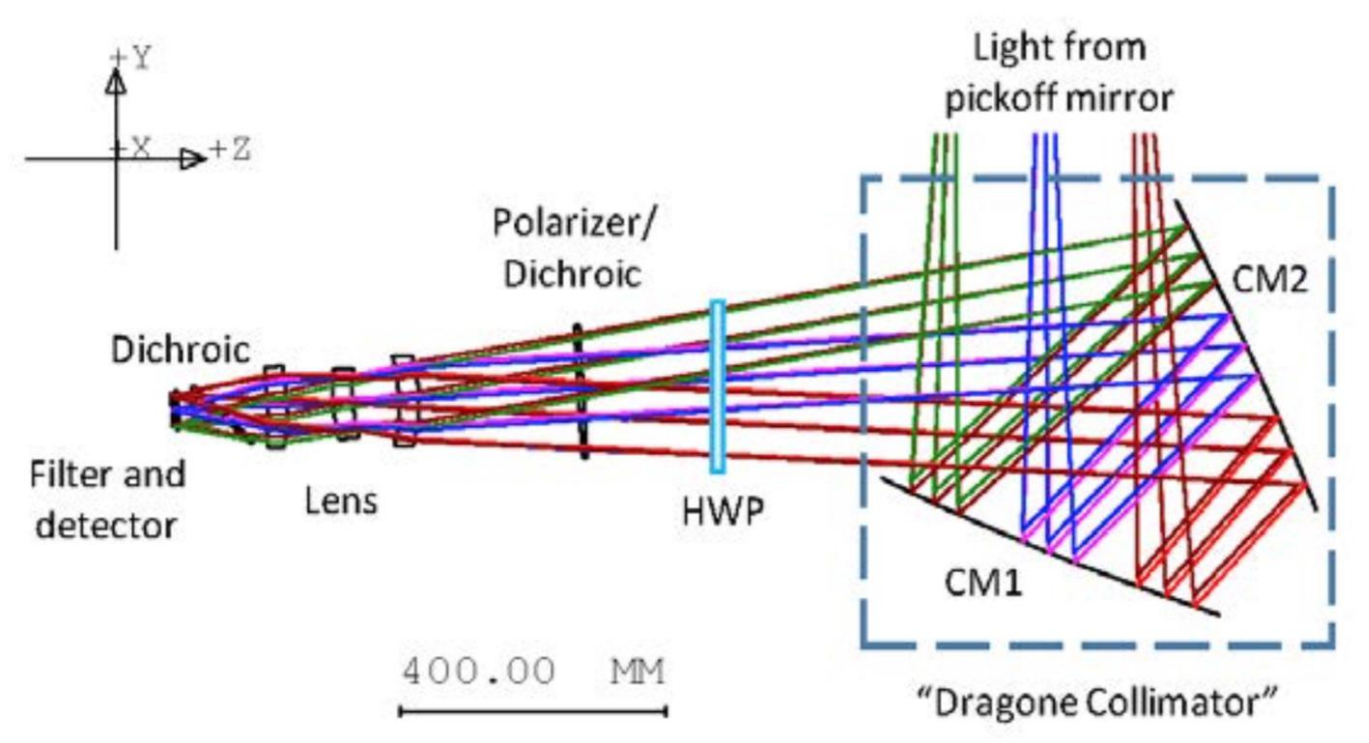

Figure 2. The FIP optical layout has heritage from SOFIA/ HAWC+.

Light is directed into the FIP instrument box by a pickoff mirror located near the telescope focal surface. Once inside the instrument box, a Dragone collimator comprising a pair of free-form (polynomial surface) mirrors (CM1, CM2), is used to collimate the light and correct aberrations. Depending on the mode of operation, the collimated beam either traverses or bypasses a half-wave plate (HWP) before passing through a dichroic or polarizer. The path depends on the waveband of interest, with a total of three possible bands: 1) short, 50100 $\mu \mathrm{m}, 2$ ) long, $250500 \mu \mathrm{m}$, and 3) the full band. If either the short or long band is used, the light passes through one of two HWPs (one for each band) and then through a polarizer. If the full band is used, the light does not 
pass through an HWP and is incident upon a dichroic rather than a polarizer. In both cases, light is split into two orthogonal paths: reflection and transmission.

The rays from these two beam paths are imaged by separate lens systems. In each case, the converging light is split in two again before passing through a filter and onto a detector plane, bringing the total number of beam paths (and detectors) to four. Like the HWP and polarizer/dichroic, the lens system switches in and out during operation depending on the waveband. The system uses an $\mathrm{f} / 10$ or $\mathrm{f} / 2$ lens for the short and long bands, respectively, bringing the total number of possible beam paths to eight. Although each of the four channels has two possible lens configurations, for either lens, the detector does not change. The HWP, dichroic/polarizer, and lenses are all switched in and out through mechanisms.

\section{MECHANICAL DESIGN}

The FIP structure is shown in Figure 3. Mechanical parts include exterior flexure mounts, a housing frame, thin enclosure, optical bench, and brackets that hold the three main mirrors. The design also includes mechanical components associated with the internal optics and detec- tor assemblies. The design tries to minimize the mechanisms in FIP.

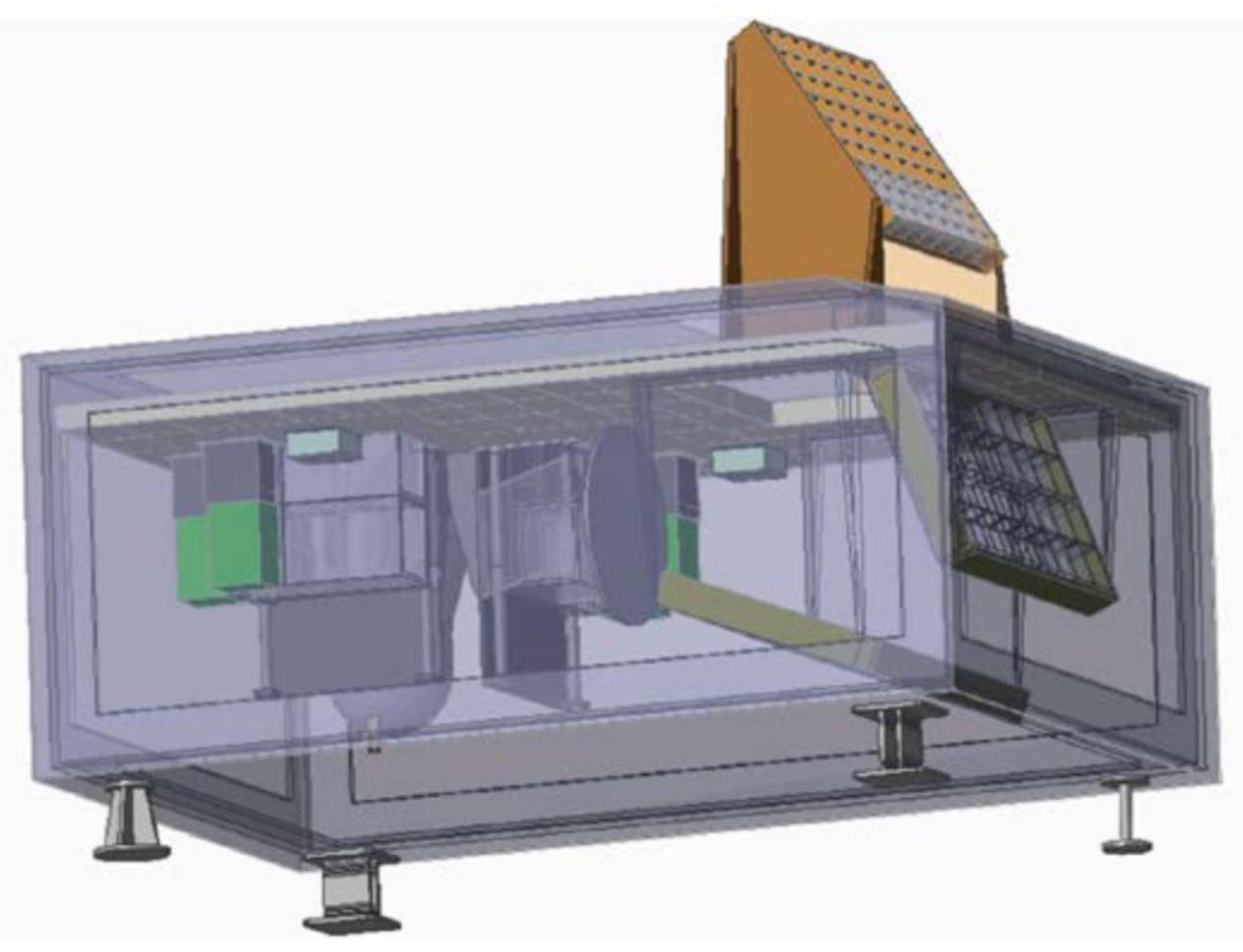

Figure 3. FIP mechanical layout. The 1 design is derived from HAWC+. Mechanical enclosures for the warm electronics are located in the spacecraft bus.

\section{DETECTORS}

On a single pixel performance basis, the detector technology required for FIP's background limited performance is well within reach: Existing laboratory detectors already meet the requirements for background-limited far-IR 
continuum $(\mathrm{R} \sim 3)$ imaging in space. A group at SRON developed low-noise $\left(N E P \sim 10^{-19} W / \sqrt{H z}\right)$ TES bolometers on suspended SiN membranes for the SAFARI instrument, with pixel pitch of $800 \mu \mathrm{m}$, comparable to the detector requirements for FIP [1]. The time constant of this device $(\tau \sim 1 \mathrm{~ms})$ also would be well suited for the high speed survey mode for OST continuum observations. Although this device had no absorber, other devices with absorbers have achieved similar sensitivity. GSFC is developing phononic filters to provide even better thermal isolation of suspended TES detectors, potentially enabling another order of magnitude improvement in sensitivity (Rostem, private communication), surpassing the FIP requirement. The GSFC team has demonstrated kilopixel Backshort Under Grid (BUG) detector arrays with SQUID readout multiplexers [2] in a relevant environment on SOFIA [3]. Alternatively, MKID detectors [4] with similar sensitivities are an option for FIP. The pixel dimensions of FIPs two concept-2 detector arrays are $120 \times 180$ for the $50 \mu \mathrm{m}$ and the $250 \mu \mathrm{m}$ bands, and $60 \times 90$ for the $100 \mu \mathrm{m}$ and the $500 \mu \mathrm{m}$ bands.

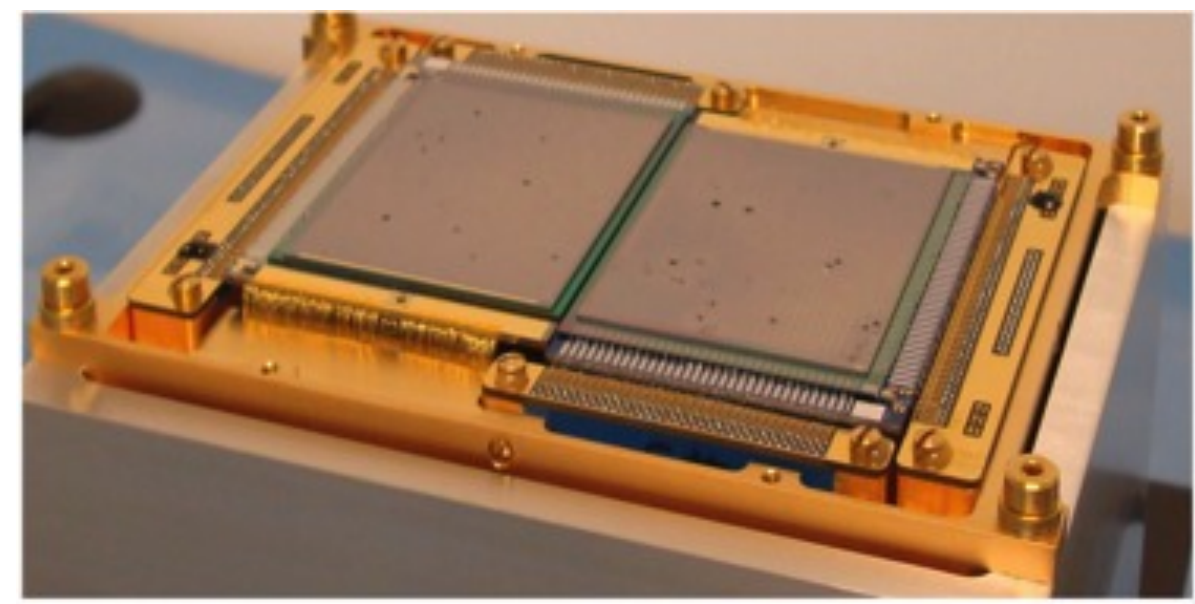

Figure 4. Photograph of one of the two focal plane arrays for SOFIAs FIR polarimeter HAWC+

\section{CRYOGENIC SQUID MULTIPLEXERS}

$\mu$-Wave SQUIDs [5] are currently the baseline technology used for the readout of the TES detectors. All elements for this scheme have been demonstrated. The channel spacing of those is not optimized for desired low-power readout. We currently plan to take this "power hit", since a possible hybrid scheme, which combines Time Division Multiplexers with uWave multiplexers [6], has too low of a Technical Readiness Level (TRL) at this time.

\section{CONCLUSION}

FIP is the background limited far-infrared continuum imager and polarimeter for the OST space telescope. The "concept-2" version of FIP provides:

- 4 far-infrared bands : 50, 100, 250, $500 \mu \mathrm{m}$

- 2 simultaneous total power bands and 2 simultaneous polarimetry bands

- Background limited detectors with $\mathrm{NEP}=2 x 10^{-19} \mathrm{~W} / \sqrt{\mathrm{Hz}}$. Additionally, higher background TES might be integrated into each pixel.

- A total of 32,500 pixels in the two detector arrays.

- Well established heritage, since similar to HAWC+ on SOFIA 


\section{REFERENCES}

[1] Suzuki, T., Khosropanah, P., Ridder, M. L., et al., 2016, Journal of Low Temperature Physics, 184, 52

[2] Allen, C.A., Benford, D.J., Miller, T.M., Moseley, S.H., Staguhn, J.G., Wollack, E.J., 2007, SPIE, 6678, 06

[3] Staguhn, J. G., Benford, D. J., Dowell, C. D., et al., 2016, Journal of Low Temperature Physics, 184, 811

[4] Baselmans, J. J. A., Bueno, J., Yates, et al., 2017, A\&A 601, A89

[5] Irwin, K. D., \& Lehnert, K. W. 2004, Applied Physics Letters, 85, 2107

[6] Reintsema, C.D., Beall, J. Doriese, W., et al., 2008, Journal of Low Temperature Physics, 151, 927 